\title{
Disparities in PET Imaging for Prostate Cancer at a Tertiary Academic Medical Center
}

\author{
Matthew D. Bucknor ${ }^{1}$, Daphne Y. Lichtensztajn ${ }^{2}$, Tracy K. Lin ${ }^{3}$, Hala T. Borno ${ }^{4}$, Scarlett L. Gomez ${ }^{2}$, and \\ Thomas A. Hope ${ }^{1}$ \\ ${ }^{I}$ Department of Radiology and Biomedical Imaging, University of California San Francisco, San Francisco, California; ${ }^{2}$ Department \\ of Epidemiology and Biostatistics, University of California San Francisco, San Francisco, California; ${ }^{3}$ Institute for Health and Aging, \\ Department of Social and Behavioral Sciences, University of California San Francisco, San Francisco, California; and ${ }^{4}$ Division of \\ Hematology/Oncology, Department of Medicine, University of California San Francisco, San Francisco, California
}

The purpose of this study was to evaluate differences between patients receiving ${ }^{18} \mathrm{~F}$-fluciclovine and ${ }^{68} \mathrm{Ga}$-prostate-specific membrane antigen ( $\left.{ }^{68} \mathrm{Ga}-\mathrm{PSMA}-11\right)$ for biochemically recurrent prostate cancer at a tertiary medical center. Methods: All ${ }^{18} \mathrm{~F}$-fluciclovine and ${ }^{68} \mathrm{Ga}-\mathrm{PSMA}-11 \mathrm{PET}$ studies performed at the University of California San Francisco from October 2015 to January 2020 were reviewed. Age, race/ethnicity, primary language, body mass index, insurance type, and home address were obtained through the electronic medical record. A logistic regression model was used to evaluate the predictor variables. Results: In total, 1,502 patients received ${ }^{6}{ }^{8} \mathrm{Ga}-\mathrm{PSMA}-11$ and 254 patients received ${ }^{18} \mathrm{~F}$-fluciclovine. Black patients had increased odds of receiving imaging with ${ }^{18} \mathrm{~F}$-fluciclovine versus ${ }^{68} \mathrm{Ga}-\mathrm{PSMA}-11$ compared with non-Hispanic White patients (odds ratio, 3.88; $95 \% \mathrm{Cl}$, 1.90-7.91). There were no other statistically significant differences. Conclusion: In patients receiving molecular imaging for prostate cancer at a single U.S. tertiary medical center, access to ${ }^{68}$ Ga-PSMA-11 for Black patients was limited, compared with non-Hispanic White patients, by a factor of nearly 4 .

Key Words: health disparities; prostate cancer; PET; ${ }^{88} \mathrm{Ga}-\mathrm{PSMA}-$ $11 ;{ }^{18} \mathrm{~F}$-fluciclovine

J Nucl Med 2021; 62:695-699

DOI: $10.2967 /$ jnumed.120.251751

$\mathbf{P}$ rostate cancer is the most common cancer in men in the United States and the second most common cause of cancer death. In 2020, an estimated 191,930 new cases of prostate cancer were diagnosed in the United States and 33,330 men died from the disease (1). In May 2016, the U.S. Food and Drug Administration (FDA) approved the use of ${ }^{18} \mathrm{~F}$-fluciclovine PET/CT imaging to evaluate disease burden in patients with suspected biochemically recurrent or persistent prostate cancer; this molecular imaging agent was covered for Medicare patients starting in 2017 (2). ${ }^{18} \mathrm{~F}$-fluciclovine is a synthetic amino acid that is not metabolized or incorporated into proteins. It targets the transmembrane amino acid transporters ACST2 and LAT1, which are overexpressed by

Received Jun. 16, 2020; revision accepted Aug. 19, 2020.

For correspondence or reprints contact: Matthew D. Bucknor, Department of Radiology and Biomedical Imaging, University of California San Francisco, 185 Berry St., Lobby 6, Suite 350, San Francisco, CA 94107.

E-mail: matthew.bucknor@ucsf.edu

Published online Sep. 25, 2020.

COPYRIGHT (C) 2021 by the Society of Nuclear Medicine and Molecular Imaging. prostate cancer cells (3). It is better able to detect metastatic disease than conventional imaging (abdominal and pelvic CT, pelvic MRI, and skeletal scintigraphy with ${ }^{99 \mathrm{~m} T c-l a b e l e d ~ m e t h y l e n e-~}$ diphosphonate) or choline PET/CT $(4,5)$.

Prostate-specific membrane antigen (PSMA) is overexpressed on prostate cancer cells, and radiotracers targeting this antigen are increasingly used to evaluate disease extent in patients with prostate cancer (6). The most commonly used PSMA-targeted radiotracer is ${ }^{68} \mathrm{Ga}-\mathrm{PSMA}-11$. Importantly, recent studies have shown that ${ }^{68}$ Ga-PSMA-11 offers significantly improved detection rates compared with ${ }^{18} \mathrm{~F}$-fluciclovine $(7,8)$. However, ${ }^{68} \mathrm{Ga}$-PSMA-11 is not yet FDA-approved and has been accessible in the United States only through clinical trials through a cost-recovery mechanism.

Health disparities in patients with prostate cancer by race/ ethnicity are well established $(9,10)$. The incidence and mortality rates of prostate cancer are significantly higher in men of African ancestry than in men from other population groups in the United States, the Caribbean, the United Kingdom, and parts of South America. Many interrelated factors have been noted as contributory, including differences in socioeconomic status and lifestyle exposures, access to health care, racial and ethnic discrimination, language and cultural barriers, and delayed disease diagnosis in socioeconomically deprived communities. The purpose of this study was to evaluate demographic differences between patients receiving ${ }^{18} \mathrm{~F}$-fluciclovine $\mathrm{PET} / \mathrm{CT}$ imaging and patients receiving ${ }^{68}$ Ga-PSMA-11 PET/CT imaging for prostate cancer at a tertiary academic medical center, in order to identify potential disparities in access to state-of-the-art care in the intermediary steps of health-care delivery-disparities that may contribute to differences in prostate cancer health outcomes.

\section{MATERIALS AND METHODS}

\section{Patient Population}

The study was approved by the institutional review board, and the need for written informed consent was waived. All ${ }^{18} \mathrm{~F}$-fluciclovine and ${ }^{68} \mathrm{Ga}$-PSMA-11 imaging studies performed at a single tertiary academic medical center between October 2015 and January 2020 were identified through a comprehensive search of the radiology report database. Patients undergoing ${ }^{68} \mathrm{Ga}$-PSMA-11 PET imaging studies were enrolled in 5 separate prospective imaging trials performed under a cost-recovery mechanism (NCT02611882, NCT02918357, NCT02919111, NCT03353740, and NCT03803475). The cost-recovery mechanism is a mechanism provided by the FDA to allow for charging of patients or insurance companies for the direct cost associated with the manufacturing of a drug. The charge associated with 
cost recovery at our institution ranged between $\$ 900$ and $\$ 1,400$, depending on the number of syntheses performed in a year.

Race/ethnicity (Asian American or Native Hawaiian/other Pacific Islander, Black or African American, Hispanic, Non-Hispanic White, or unknown), primary language (English or not English), body mass index, primary insurance payor (commercial, government, or unknown), and home address were obtained through the electronic medical record database. Duplicate patient records within each category of imaging study were removed. Maps depicting the distribution of patient ZIP codes were created for those receiving each of the 2 radiotracers through Google My Maps software (Fig. 1).

\section{Statistical Analysis}

Demographic characteristics were summarized for each patient cohort. Home addresses were geocoded to census block groups and assigned to a tertile of neighborhood socioeconomic status (nSES) using a previously described composite measure based on statewide distribution (11). Demographic percentages were calculated for each group. The association of each demographic variable with use of ${ }^{18} \mathrm{~F}$ fluciclovine versus ${ }^{68} \mathrm{Ga}$-PSMA-11 was modeled using a multivariate logistic regression analysis. All demographic variables were selected a priori on the basis of potential for related referral bias, and all were included in the multivariable model. For patients missing specific demographic information, the available covariates were entered into the model. All analyses were performed using Stata, and $P$ values of less than 0.05 were considered statistically significant.

\section{RESULTS}

In total, 1,756 patients were included in the study, including 1,502 patients who received ${ }^{68} \mathrm{Ga}$-PSMA-11 and 254 patients who received ${ }^{18} \mathrm{~F}$-fluciclovine (Table 1 ). Non-Hispanic White patients made up $78.8 \%$ of the entire study population $(1,383 / 1,756)$, and minorities composed $21.2 \%(373 / 1,756) ; 66.6 \%$ of patients

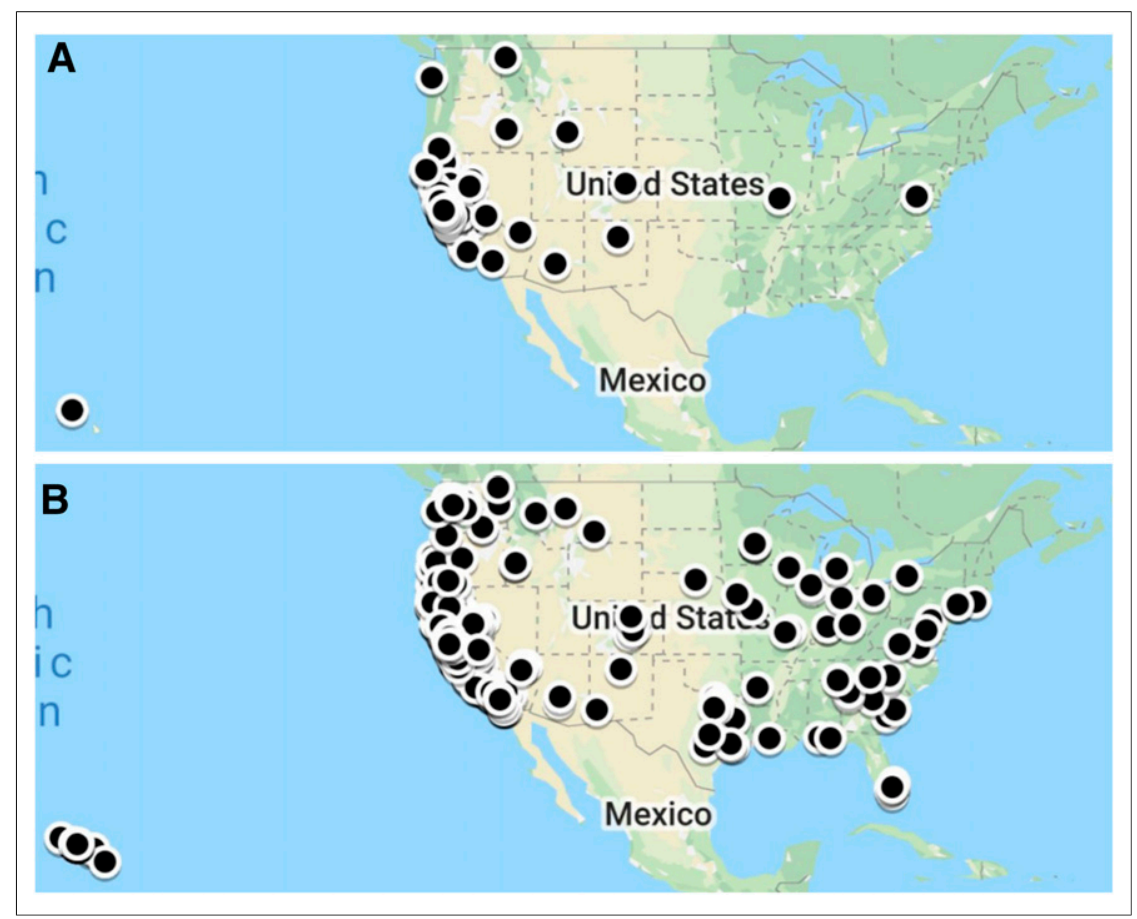

FIGURE 1. Mapped distribution of ZIP codes of patients receiving ${ }^{18} \mathrm{~F}$-fluciclovine $(\mathrm{A})$ or ${ }^{68} \mathrm{Ga}-$ PSMA-11 (B). were from the highest tertile of nSES $(998 / 1,498)$, followed by the middle tertile $(24.7 \%, 370 / 1,498)$, and the lowest tertile $(8.7 \%$, 130/1,498). Regarding the intersection of race/ethnicity and nSES, Black patients were the lowest percentage in the highest nSES tertile $(40.0 \%, 16 / 40)$ and the highest percentage in the lowest tertile $(27.5 \%, 11 / 40)$ (Table 2). Hispanic patients were the next lowest percentage in the highest nSES tertile $(51.0 \%, 26 / 51)$ and the next highest percentage in the lowest tertile $(25.5 \%, 13 / 51)$.

Age was similar among patients who received ${ }^{18} \mathrm{~F}$-fluciclovine (mean, $69.8 \pm 7.9 \mathrm{y}$ ) and ${ }^{68} \mathrm{Ga}$-PSMA-11 (mean, $69.6 \pm 7.7 \mathrm{y}$ ). BMI was also similar between the 2 groups. Relatively few patients in the study did not indicate English as their preferred language, with non-English speakers making up 3.1\% of the ${ }^{18} \mathrm{~F}$-fluciclovine group (8/254) and $1.9 \%$ of the ${ }^{68}$ Ga-PSMA-11 group $(28 / 1,502)$.

As seen in Table 1, Black patients had approximately 4 times the odds of receipt of ${ }^{18} \mathrm{~F}$-fluciclovine imaging that ${ }^{68} \mathrm{Ga}$-PSMA11 had (OR, 3.88; 95\% CI, 1.90-7.91). There were no significant differences in other patient demographics between the 2 groups. A trend was noted toward increased odds of imaging with ${ }^{18} \mathrm{~F}$-fluciclovine compared with ${ }^{68}$ Ga-PSMA-11 for patients identifying as Asian American or Native Hawaiian/other Pacific Islander (odds ratio, 1.64; 95\% CI, 0.95-2.85; $P=0.073$ ). Patients with government insurance and increased age also showed trends toward increased odds of imaging with ${ }^{18} \mathrm{~F}$-fluciclovine, but the magnitude of the differences was relatively small.

\section{DISCUSSION}

This study found differential use patterns for molecular imaging modalities by race/ethnicity. Although both ${ }^{18} \mathrm{~F}$-fluciclovine and ${ }^{68}$ Ga-PSMA-11 have dramatically improved the ability to evaluate overall disease burden in patients with prostate cancer, ${ }^{68} \mathrm{Ga}-$ PSMA-11 has emerged as the superior tracer, with higher sensitivity (7). The current study demonstrates that when available through clinical trials, access to ${ }^{68} \mathrm{Ga}$-PSMA-11 PET was significantly limited for Black patients, compared with non-Hispanic White patients, by a factor of nearly 4.

The ways in which crucial intermediary steps in health-care delivery between patient presentation and health-care outcomes, such as advanced diagnostic imaging, contribute to disparities in health outcomes are poorly understood across many disease types and imaging modalities. Disparities in healthcare imaging have been previously described, somewhat extensively in the field of mammography, and more generally with regard to the frequency of missed care opportunities, as well as differences in wait times to receive advanced imaging (12-18). A more recent study of the follow-up of incidental findings on abdominal imaging demonstrated that elderly patients and emergency department patients were less likely to complete follow-up imaging and that insurance status and race may also contribute to differences in follow-up rates (19). However, the literature examining disparities in radiology remains sparse. The current study demonstrates 
TABLE 1

Demographic Characteristics of Patients Undergoing ${ }^{18} \mathrm{~F}-\mathrm{Fluciclovine} \mathrm{Versus}{ }^{68} \mathrm{Ga}-\mathrm{PSMA}-11$ for Prostate Cancer and Results of Multivariate Regression Analysis

\begin{tabular}{|c|c|c|c|c|}
\hline Characteristic & ${ }^{18} \mathrm{~F}$-fluciclovine & ${ }^{68} \mathrm{Ga}-\mathrm{PSMA}$ & Odds ratio & $P$ \\
\hline All patients & 254 & 1,502 & & \\
\hline Age (mean $\pm \mathrm{SD}$ ) & $69.8 \pm 7.9$ & $69.6 \pm 7.7$ & $1.01(0.99-1.04)$ & 0.097 \\
\hline Body mass index (mean \pm SD) & $28.1 \pm 4.0$ & $27.7 \pm 4.5$ & $1.00(0.97-1.04)$ & 0.761 \\
\hline \multicolumn{5}{|l|}{ Race } \\
\hline Non-Hispanic White & $182(71.6 \%)$ & $1201(80.0 \%)$ & 1.00 & \\
\hline Black or African American & $17(6.7 \%)$ & $24(1.6 \%)$ & $3.88(1.90-7.91)$ & $<0.001$ \\
\hline Hispanic & $13(5.1 \%)$ & $45(3.0 \%)$ & $1.79(0.84-3.81)$ & 0.131 \\
\hline $\begin{array}{l}\text { Asian American or Native Hawaiian/other } \\
\text { Pacific Islander }\end{array}$ & $22(8.7 \%)$ & $87(5.8 \%)$ & $1.64(0.95-2.85)$ & 0.073 \\
\hline Unknown & $20(7.9 \%)$ & $145(9.6 \%)$ & $1.06(0.55-2.02)$ & 0.87 \\
\hline \multicolumn{5}{|l|}{ Preferred language } \\
\hline English & $246(96.9 \%)$ & $1474(98.1 \%)$ & 1.00 & \\
\hline Not English & $8(3.1 \%)$ & $28(1.9 \%)$ & $1.29(0.53-3.13)$ & 0.581 \\
\hline \multicolumn{5}{|l|}{ Insurance type } \\
\hline Commercial & $87 / 238(36.6 \%)$ & $478(31.8 \%)$ & 1.00 & \\
\hline Government & $149 / 238(62.6 \%)$ & 929 (61.9\%) & $0.70(0.48-1.01)$ & 0.06 \\
\hline Unknown & $2 / 238(0.8 \%)$ & $95(6.3 \%)$ & $0.39(0.09-1.73)$ & 0.215 \\
\hline \multicolumn{5}{|l|}{ nSES } \\
\hline Highest tertile & $143 / 235$ (60.9\%) & $855 / 1,263(67.7 \%)$ & 1.00 & \\
\hline Middle tertile & $67 / 235(28.5 \%)$ & $303 / 1,263$ (24.0\%) & $1.27(0.89-1.81)$ & 0.194 \\
\hline Lowest tertile & $25 / 235$ (10.6\%) & $105 / 1,263(8.3 \%)$ & $1.08(0.62-1.91)$ & 0.78 \\
\hline
\end{tabular}

${ }^{\star}$ For any categories in which demographic information was missing from patient records, new denominator is noted (insurance type and nSES).

Data in parentheses are percentage or $95 \% \mathrm{Cl}$.

the essential need for more studies of this kind in radiology as a critical precondition for developing policies and procedures that can identify and eliminate structural barriers to equitable care delivery.

The exact factors that contributed to disparities in use of imaging in this study are unclear. As with disparities in prostate cancer health outcomes, different aspects of social determinants of health may play a role. Our nSES index included a range of variables, including multiple measures of education, income, employment, and housing. The study population was more affluent than average, with $66.6 \%$ of patients belonging to the highest statewide nSES tertile, and there was also a higher percentage of non-Hispanic White patients $(78.8 \%)$ than found in regional census data (52.5\%) (20). nSES was not a significant covariate in our analysis, suggesting that additional factors contributed to the disparities that were observed. However, fewer Black patients $(40.0 \%)$ than non-Hispanic White patients $(68.6 \%)$ belonged to the highest nSES tertile, and a larger percentage of Black patients belonged to a lower nSES tertile than any other group $(27.5 \%)$. The combined effect of these demographic patterns may have contributed to our results in ways that could not be directly demonstrated through our specific regression analysis. Additionally, of note, a recent study on the use of ${ }^{18} \mathrm{~F}$ fluciclovine in a separate tertiary academic medical center suggested possible under-referrals of Black patients for molecular imaging, and similar trends may have occurred at our medical center.

TABLE 2

Distribution of nSES by Race/Ethnicity

\begin{tabular}{lccc}
\hline \multicolumn{1}{c}{ Race/ethnicity } & Highest tertile & Middle tertile & Lowest tertile \\
\hline All patients & 998 & 370 & 130 \\
Non-Hispanic White & $808(68.6 \%)$ & $277(23.5 \%)$ & $93(7.9 \%)$ \\
Black or African American & $16(40.0 \%)$ & $13(32.5 \%)$ & $11(27.5 \%)$ \\
Hispanic & $26(51.0 \%)$ & $12(23.5 \%)$ & $13(25.5 \%)$ \\
Asian American or Native Hawaiian/other Pacific Islander & $74(74.0 \%)$ & $23(23.0 \%)$ & $3(3.0 \%)$ \\
Unknown & $74(57.4 \%)$ & $45(34.9 \%)$ & $10(7.7 \%)$ \\
\hline
\end{tabular}


More work is needed to better understand the complex social and regulatory factors, including unconscious bias, that may influence imaging access (21).

Although, historically, both imaging agents have been used for similar prostate cancer populations at the study institution, the most glaring difference between the routine use of each of these agents is that ${ }^{68} \mathrm{Ga}$-PSMA-11 has been available only through research trials whereas ${ }^{18} \mathrm{~F}$-fluciclovine is covered by both government and commercial payors, secondary to its FDA approval in 2016. Remarkably, despite the requirement for study participation and the possibility of self-pay, nearly 6 times as many patients in this study were imaged with ${ }^{68} \mathrm{Ga}$-PSMA-11 as with ${ }^{18} \mathrm{~F}$-fluciclovine. Thus, the current study's findings highlight potential disparities in access to imaging research trials for Black patients. This problem is common to many clinical trials but often can be difficult to detect, particularly in radiology trials, as many enroll relatively fewer patients (22). As we improve our understanding of how bias and racism may limit health-care access, more work is needed to develop enrollment strategies designed to promote equitable recruitment, including novel digital marketing interventions, revised screening processes, and sliding-scale financial reimbursement.

Although meaningful conclusions can be drawn from this study, there are several limitations worth noting. For one, this was a singlesite retrospective study; therefore, the study sample may not be representative of all patients for whom molecular imaging may be considered. The regression analysis did not adjust for disease attributes such as Gleason score or prostate-specific antigen laboratory value. However, the current institution-specific practice is that both of these modalities are applied similarly, and the use of exhaustive and relatively large study cohorts helps to mitigate this potential confounder. Additionally, differences in individual drivers of nSES, such as income or wealth, may have contributed to the differences that we identified in our study if obscured by the larger nSES composite.

Despite these limitations, this study had several strengths. It had a large sample size, which increased the ability to detect differences between the characteristics of patients who received each type of imaging study. The clear discrepancy highlights the need for more innovative and equitable recruitment strategies, as well as the important role that government regulatory agencies can potentially play in facilitating health equity. Data from ${ }^{68} \mathrm{Ga}-$ PSMA-11 PET trials is currently under FDA review, and potential approval in the near future could increase the availability of this drug to more patients.

Finally, at many institutions, ${ }^{68} \mathrm{Ga}-\mathrm{PSMA}-11$ PET has been available only in clinical trials through a cost-recovery mechanism, by which the FDA allows centers to pay for the cost of the radiotracer if no corporate entity is developing the drug (23). As ${ }^{68} \mathrm{Ga}$-PSMA-11 was not patented, academia took the initiative in development and most trials were performed under cost recovery. Through this mechanism, patients often would be financially liable for the direct cost of the radiotracer and possibly the cost of the technical component of the PET imaging, which could pose a significant barrier to low-income groups. Additionally, there may be variability in how individual institutions approved cost recovery across institutions.

\section{CONCLUSION}

The current study demonstrated that at a single tertiary medical center, access to ${ }^{68} \mathrm{Ga}$-PSMA-11 PET/CT was significantly limited for Black patients, compared with non-Hispanic White patients, by a factor of nearly 4 . Disparities in access to research trials and concerns about potential financial burden from the cost-recovery mechanism may have contributed to differences in imaging rates. More studies evaluating potential disparities in use of imaging technologies, related to known social determinants of health, are essential for building equitable systems of healthcare delivery.

\section{DISCLOSURE}

No potential conflict of interest relevant to this article was reported.

\section{KEY POINTS}

QUESTION: Are there disparities in PET imaging access for patients with suspected biochemically recurrent or persistent prostate cancer?

PERTINENT FINDINGS: A multivariate logistic regression analysis of 1,756 patients who received ${ }^{68} \mathrm{Ga}-\mathrm{PSMA}-11$ or ${ }^{18} \mathrm{~F}-$ fluciclovine PET imaging for prostate cancer at a tertiary academic medical center in the United States demonstrated that access to ${ }^{8} \mathrm{Ga}-\mathrm{PSMA}-11$ for Black patients was limited, compared with non-Hispanic White patients, by a factor of nearly 4 .

IMPLICATIONS FOR PATIENT CARE: Disparities in the use of PET imaging may contribute to disparities in health outcomes, and more work is needed to better understand causative factors, including the role of bias and access to clinical trials.

\section{REFERENCES}

1. Cancer facts \& figures 2020. American Cancer Society website. https://www. cancer.org/research/cancer-facts-statistics/all-cancer-facts-figures/cancer-facts-figures2020.html. Accessed December 9, 2020.

2. FDA approves new diagnostic imaging agent to detect recurrent prostate cancer. Food and Drug Administration website. https://www.fda.gov/newsevents/press-announcements/fda-approves-new-diagnostic-imaging-agentdetect-recurrent-prostate-cancer. Published May 27, 2016. Accessed December 9, 2020.

3. Parent EE, Schuster DM. Update on ${ }^{18} \mathrm{~F}$-fluciclovine pet for prostate cancer imaging. J Nucl Med. 2018;59:733-739.

4. Gusman M, Aminsharifi JA, Peacock JG, Anderson SB, Clemenshaw MN, Banks KP. Review of ${ }^{18} \mathrm{~F}$-fluciclovine PET for detection of recurrent prostate cancer. Radiographics. 2019;39:822-841.

5. Alemozaffar M, Akintayo AA, Abiodun-Ojo OA, et al. $\left[{ }^{18} \mathrm{~F}\right]$ fluciclovine PET/CT for preoperative staging in patients with intermediate to high-risk primary prostate cancer. J Urol. 2020;204:734-740.

6. Schwarzenboeck SM, Rauscher I, Bluemel C, et al. PSMA ligands for PETimaging of prostate cancer. J Nucl Med. 2017;58:1545-1552.

7. Calais J, Ceci F, Eiber M, et al. ${ }^{18}$ F-fluciclovine PET-CT and ${ }^{68}$ Ga-PSMA- 11 PET-CT in patients with early biochemical recurrence after prostatectomy: a prospective, single-centre, single-arm, comparative imaging trial. Lancet Oncol. 2019;20:1286-1294.

8. Calais J, Fendler W, Herrmann K, et al. Head-to-head comparison of ${ }^{68} \mathrm{Ga}-$ PSMA-11 PET/CT and ${ }^{18} \mathrm{~F}$-flucilovine PET/CT in a case series of 10 patients with prostate cancer recurrence. J Nucl Med. 2018;59:789-794.

9. Riviere P, Luterstein E, Kumar A, et al. Survival of African American and nonHispanic white men with prostate cancer in an equal-access health care system. Cancer. 2020;126:1683-1690.

10. Borno H, George DJ, Schnipper LE, Cavalli F, Cerny T, Gillessen S. All men are created equal: addressing disparities in prostate cancer care. Am Soc Clin Oncol Educ Book. 2019;39:302-308.

11. Yang J, Schupp CW, Harrati A, Clarke C, Keegan THM, Gomez SL. Developing an Area-Based Socioeconomic Measure from American Community Survey Data. Cancer Prevention Institute of California; 2014. 2014:1-17. 
12. Rauscher GH, Allgood KL, Whitman S, Conant E. Disparities in screening mammography services by race/ethnicity and health insurance. $J$ Womens Health (Larchmt). 2012;21:154-160.

13. Peek ME, Han JH. Disparities in screening mammography. J Gen Intern Med. 2004;19:184-194.

14. Press R, Carrasquillo O, Sciacca RR, Giardina E-GV. Racial/ethnic disparities in time to follow-up after an abnormal mammogram. J Womens Health (Larchmt). 2008;17:923-930.

15. Khan-Gates JA, Ersek JL, Eberth JM, Adams SA, Pruitt SL. Geographic access to mammography and its relationship to breast cancer screening and stage at diagnosis: a systematic review. Womens Health Issues. 2015;25:482-493.

16. Lee B, Otero HJ, Whitehead MT. The fate of radiology report recommendations at a pediatric medical center. Pediatr Radiol. 2017;47:1724-1729.

17. Glover M, Daye D, Khalilzadeh O, et al. Socioeconomic and demographic predictors of missed opportunities to provide advanced imaging services. J Am Coll Radiol. 2017;14:1403-1411.
18. Daye D, Carrodeguas E, Glover MIV, Guerrier CE, Harvey HB, Flores EJ. Impact of delayed time to advanced imaging on missed appointments across different demographic and socioeconomic factors. J Am Coll Radiol. 2018;15:713-720.

19. Cho JK, Zafar HM, Lalevic D, Cook TS. Patient factor disparities in imaging follow-up rates after incidental abdominal findings. AJR. 2019;212:589-595.

20. Home page. Bay Area Census website. http://www.bayareacensus.ca.govbayarea. htm. Accessed December 9, 2020.

21. Galgano SJ, Calderone CE, McDonald AM, et al. Patient demographics and referral patterns for [F-18]fluciclovine-Pet imaging at a tertiary academic medical center. J Am Coll Radiol. 2019;16:315-320.

22. Borno HT, Bakke BM, Kaplan C, et al. A step towards equitable clinical trial recruitment: a protocol for the development and preliminary testing of an online prostate cancer health information and clinical trial matching tool. Pilot Feasibility Stud. 2019;5:123-128.22.

23. Van Norman GA. Expanding patient access to investigational drugs: single patient investigational new drug and the "right to try." JACC Basic Transl Sci. 2018;3:280-293. 